\title{
Voltammetric behaviour of biological macromolecules at arrays of
}

\section{aqueous | organogel micro-interfaces}

\author{
Micheál D. Scanlon, Jörg Strutwolf and Damien W. M. Arrigan*, ${ }^{1}$ \\ Tyndall National Institute, Lee Maltings, University College Cork, Cork, Ireland. \\ *Corresponding author: Email: d.arrigan@curtin.edu.au; \\ fax: +61-8-92664699; phone: +61-8-92669735.
}

\begin{abstract}
The behaviour of two biological macromolecules, bovine pancreatic insulin and hen-eggwhite lysosyme (HEWL), at aqueous-organogel interfaces confined within an array of solidstate membrane micropores was investigated via cyclic voltammetry (CV). The behaviour observed is discussed in terms of possible charge transferring species and mass transport in the interfacial reaction. Comparison of CV results for HEWL, insulin, and the wellcharacterised model ion tetraethylammonium cation $\left(\mathrm{TEA}^{+}\right)$revealed that the biomacromolecules undergo an interfacial reaction comprising biomacromolecular adsorption and facilitated transfer of electrolyte anions from the organic phase to a protein layer on the aqueous side of the interface, whereas $\mathrm{TEA}^{+}$undergoes a simple ion transfer process. Evidence for biomacromolecular adsorption on the aqueous side of the micro-interfaces is provided by comparison of the CVs for $\mathrm{TEA}^{+}$ion transfer in the presence and absence of the biomacromolecules. Similar experiments in the presence of the low generation polypropylenimine tetraamine dendrimer, (DAB-AM-4), a smaller synthetic molecule, revealed it to be non-adsorbing. The behaviour of biological macromolecules at miniaturised
\end{abstract}

\footnotetext{
${ }^{1}$ Present address: Nanochemistry Research Institute, Curtin University of Technology, GPO Box U1987, Perth, WA 6845, Australia. Email: d.arrigan@curtin.edu.au, fax: +61-8-92664699; phone: +61-8-92669735.
} 
Revised Paper Ref.: C003323E. PCCP (RSC).

aqueous-organogel interfaces involves adsorption on the aqueous side of the interface and transfer of organic phase electrolyte anions across the interface to associate with the adsorbed biomacromolecule. The data presented support the previously suggested mechanism for biomacromolecular voltammetry at liquid-liquid interfaces, involving adsorption and facilitated ion-transfer of organic electrolyte anions.

\section{Introduction}

The voltammetric behaviour of biological macromolecules has been studied at the interface between two immiscible electrolyte solutions (ITIES) as a means to detection of such molecules and evaluation of their behaviours. The bioanalytical capabilities of electrochemistry at the ITIES ${ }^{1-6}$ offers scope for the detection of non-redoxactive ions or for the detection of ions suffering from interference when detected by oxidation or reductionbased methods. Thus the detection of biomolecules such as dopamine, ${ }^{7-9}$ amino acids, ${ }^{10}$ heparin, ${ }^{11-13}$ protamine, ${ }^{14,15}$ and drug molecules ${ }^{16,17}$ have been reported. From our own laboratory, a range of biological macromolecules have been studied, namely haemoglobin $(\mathrm{Hb}),{ }^{18,19}$ bovine pancreatic insulin, ${ }^{20}$ and hen-egg-white lysozyme (HEWL), ${ }^{21}$ as part of ongoing investigations into the use of electrochemical methods at liquid-liquid interfaces as the basis for label-free bioanalytical detection methods. ${ }^{1}$ In the proposed mechanisms for the electrochemical responses of haemoglobin, ${ }^{18,19}$ insulin, ${ }^{20}$ and $\mathrm{HEWL}^{21}$ at the ITIES, the organic phase electrolyte anion, typically tetrakis(4-chlorophenyl)borate anion ( $\left.\mathrm{TPBCl}^{-}\right)$, is believed to play a significant role. It was proposed that the Hb, insulin, and HEWL molecules adsorb on the aqueous side of the ITIES and then facilitate the transfer of $\mathrm{TPBCl}^{-}$across the interface. The biomacromolecules may thus be equated to an "ionophore" that facilitates the transfer of the $\mathrm{TPBCl}^{-}$ion. 
This article focuses on investigations into the voltammetric behaviour of insulin and HEWL at miniaturised liquid-liquid interfaces. Arrays of micron-sized ITIES ( $\mu$ ITIES) were formed within the confines of silicon solid-state micropore arrays. ${ }^{22}$ The organic phase electrolyte solution is typically present as an organogel that, due to the hydrophobic nature of the pore walls, fills the pores thereby establishing inlaid $\mu$ ITIES arrays on the aqueous side of the membrane. ${ }^{23}$ Furthermore, the use of an organo-gel stabilises the soft interface between the two phases. Thus far, the diffusion of the biomacromolecule in the aqueous phase to the pore mouth has been assumed to be the controlling step in the electrochemical detection mechanism. The enhanced mass transport at the $\mu$ ITIES array is expected to yield improvements in both the limits of detection (LOD) and sensitivity of the analytical response for biomacromolecules in comparison to those achieved at the regularly-sized ITIES (typically millimeter-sized interfaces) under otherwise identical experimental conditions. This strategy has previously been pursued with regard to the low-level detection of smaller biomolecules such as a selection of oligopeptides ${ }^{24}$ and a $\beta$-blocker drug. ${ }^{25}$

By comparison of experimental and simulated voltammograms, Strutwolf et al. ${ }^{23}$ have identified specific $\mu$ ITIES array designs capable of establishing asymmetric diffusion fields for the simple reversible transfer of a model ion (tetraethylammonium cation $\left(\mathrm{TEA}^{+}\right)$) on application of a cyclic voltammetric (CV) scan. It was found that the pores used to form such $\mu$ ITIES were filled completely with the organogel phase, so that the ITIES was located at the pore orifice on the aqueous side. Consequently, spherical diffusion was established for the transfer of the $\mathrm{TEA}^{+}$probe ion from the aqueous to the organic phase, resulting in peakless voltammograms exhibiting a steady-state limiting current. In contrast, the current of the 
Revised Paper Ref.: C003323E. PCCP (RSC).

reverse scan showed a clear peak, which was due to the $\mathrm{TEA}^{+}$ions being mainly trapped within the pores where linear diffusion dominated. The asymmetry in the diffusion in both directions across the ITIES was supported by the difference of the diffusion coefficient for $\mathrm{TEA}^{+}$in both phases: the diffusion coefficient in the viscous organic phase was reduced by a factor of ca. nine compared to that in the aqueous phase. Such a $\mu$ ITIES array design was chosen in this study with a view to utilising the asymmetric diffusion fields established via CV to reveal further details of the insulin and HEWL detection processes. For example, Zazpe et al. $^{22}$ have applied this particular $\mu$ ITIES array geometry to study the facilitated transfer of $\mathrm{K}^{+}$across the $\mu$ ITIES array, in the presence of the ionophore dibenzo-18-crown- 6 ether (DB18C6), via the transfer by interfacial complexation/transfer by interfacial decomplexation (TIC/TID) mechanism. In a manner analogous to studies of TIC/TID at a micropipettesupported ITIES, ${ }^{26,} 27$ the diffusion regimes observed at this $\mu$ ITIES array formed in a solidstate membrane are determined by the limiting factor in the transport process (i.e. the controlling step may involve either the analyte or the ionophore). Thus, mechanistic information on whether the transport process is being controlled by diffusion to the $\mu$ ITIES array of a species in either the organogel-filled micropores or the aqueous phase may be obtained.

As reported previously, ${ }^{20,}{ }^{21}$ HEWL and insulin both adsorb at the ITIES during a CV cycle. Further evidence of biomacromolecular adsorption at the ITIES was expected by conducting experiments at $\mu$ ITIES. Comparisons were made to the electrochemical response of a much smaller synthetic macromolecule that was not expected to adsorb at the $\mu$ ITIES, polypropylenimine tetraamine dendrimer, generation 1.0 (DAB-AM-4), under identical experimental conditions to those implemented for HEWL and insulin. In particular, the effects 
Revised Paper Ref.: C003323E. PCCP (RSC).

of the presence of the adsorbing biomacromolecules, versus the non-adsorbing dendrimer, on the transfer of TEA ${ }^{+}$across the micro-interfaces were examined.

\section{Experimental details}

Reagents. All reagents were purchased from Sigma Aldrich Ireland Ltd. and used as received with the exception of 1,6-dichlorohexane (1,6-DCH) which was purified as reported. ${ }^{28}$ The aqueous phase electrolytes of $10 \mathrm{mM} \mathrm{LiCl}$ and $10 \mathrm{mM} \mathrm{HCl}$ were prepared in ultrapure water (resistivity: $18 \mathrm{M} \Omega \mathrm{cm}$ ) from an Elgastat maxima-HPLC (Elga, UK). From previous studies of the insulin ${ }^{20}$ and $\mathrm{HEWL}^{21}$ electrochemical responses it was noted that the maximum peak currents for CV at ITIES on the forward sweep were observed under acidic aqueous phase conditions, with the $\mathrm{pH}$ being considerably lower than the isoelectric points (pI) of either molecule (the pIs are 11.35 and $~ 5.5$ for $\mathrm{HEWL}^{29}$ and insulin, ${ }^{30-32}$ respectively). Thus, the aqueous phase electrolyte solution chosen was $10 \mathrm{mM} \mathrm{HCl}$, with a $\mathrm{pH}$ of 2 . Some denaturation of protein is expected at $\mathrm{pH} 2,^{33,34}$ although the impact of this on the electrochemical response has to be balanced with the fact that at $\mathrm{pH}$ values close to each protein's isoelectric point no electrochemical signal is obtained. ${ }^{20,21}$ Low $\mathrm{pH}$ solutions also favour the presence of insulin in monomeric form. ${ }^{20}$ Stock solutions of HEWL and bovine pancreatic insulin were prepared in $10 \mathrm{mM} \mathrm{HCl}_{(\text {aq.) }}$ daily and stored at $+4{ }^{\circ} \mathrm{C}$. Tetraethylammonium chloride $\left(\mathrm{TEA}^{+} \mathrm{Cl}^{-}\right)$was added to the electrochemical cell following biomacromolecule experiments, in which case the $\mathrm{TEA}^{+} \mathrm{Cl}^{-}$stock solution was prepared in 10 $\mathrm{mM} \mathrm{HCl}\left(\right.$ aq.). For experiments involving ion transfer of $\mathrm{TEA}^{+}$in the absence of the biomacromolecules, the $\mathrm{TEA}^{+} \mathrm{Cl}^{-}$stock solution was prepared in $10 \mathrm{mM} \mathrm{LiCl}_{(\mathrm{aq} .)}$, which provided a large potential window. 
Revised Paper Ref.: C003323E. PCCP (RSC).

The organic phase electrolyte salt was prepared by metathesis of $10 \mathrm{mM}$ bis(triphenylphosphoranylidene)ammonium chloride, (BTPPA)(Cl), and potassium tetrakis(4chlorophenyl)borate, (K)(TPBCl), to obtain (BTPPA)(TPBCl), following the published procedure. $^{35}$ The organic reference solution consisted of $10 \mathrm{mM}(\mathrm{BTPPA})(\mathrm{Cl})$ dissolved in 10 $\mathrm{mM} \mathrm{LiCl}_{(\mathrm{aq} .)}$. The organic phase electrolyte solution for the $\mu$ ITIES array experimental setup was present as an organogel, comprising $10 \mathrm{mM}$ (BTPPA)(TPBCl) dissolved in 1,6-DCH and gellified with low molecular weight poly(vinyl chloride), (PVC), as described elsewhere. ${ }^{24}$

Electrochemical details. All electrochemical measurements were performed with a CHI 620B potentiostat (CH Instruments, Texas, USA). With the $\mu$ ITIES array, a 3-electrode cell was employed $^{23,24}$ comprising one Pt mesh counter electrode (in the aqueous phase) and two Ag/AgCl reference electrodes (one in each phase). The compositions of the different electrochemical cells implemented at the gellified $\mu$ ITIES array are summarised in Figure 1. Background-subtracted CVs were obtained using the $\mathrm{CH}$ Instruments software. All experiments were carried out using the same micropore array design which was chosen so as to have no diffusional overlap between adjacent pores in the array. ${ }^{23}$ The array contained 8 pores, with pore radii of (26.00 \pm 0.09$) \mu \mathrm{m}$ and pore centre-to-centre separations of $(985.73 \pm$ 1.50) $\mu \mathrm{m}$. These geometric parameters were determined from scanning electron microscopy (SEM) images of the array and each value was averaged over 6 pores. The geometric area of the interfaces in the $\mu$ ITIES array was obtained from the area of each pore, $2.12 \times 10^{-5} \mathrm{~cm}^{2}$, multiplied by the number of pores in the array, $N_{p}=8$, to give the geometric area, $1.7 \times 10^{-4}$ $\mathrm{cm}^{2}$.

\section{Results and discussion}


Revised Paper Ref.: C003323E. PCCP (RSC).

Simple (unassisted) ion transfer at the gellified $\mu$ ITIES array. First, CV involving only $\mathrm{TEA}^{+}$ions as the transferring species was performed, where the $\mathrm{TEA}^{+}$ions are initially present in the aqueous phase and are then transferred, under potential-scanned control, into the gellified organic phase. During the reverse scan these ions are transferred back from the organic into the aqueous phase. Figure 2(A) shows CVs recorded at scan rates between 5 and $25 \mathrm{mV} \mathrm{s}^{-1}$ in the presence of $100 \mu \mathrm{mEA}^{+}$. The asymmetry in the diffusion zones that develops during the forward and reverse scans is evident from the shapes of the CVs. The interface is inlaid with the pore orifice at the aqueous side, resulting in a spherical diffusion field and a steady state forward-scan voltammogram with a well-defined limiting current. However, the transferred TEA ${ }^{+}$ions accumulate in the micropores, as shown previously ${ }^{23}$ due to the depth of the pore and the low diffusion coefficient of the $\mathrm{TEA}^{+}$ions in the gellified organic phase. The back-transfer is thus dominated by linear diffusion, since the pore wall surpresses the contribution of radial diffusion. This situation is also clarified by Figure 2(B), which shows the dependence of the forward limiting current and the reverse peak current on the square root of the scan rate. The limiting current is almost invariant with increasing scan rate. A very slight increase in current was observed on the forward sweep, but this may be attributed to slight discrepancies which arise in the background-subtraction process with increasing scan rate. In contrast, the peak current of the reverse scan shows the behaviour expected for linear diffusion-dominated transfer. As will be shown below, the presence of insulin and HEWL induces different behaviour.

CV of insulin and HEWL at the gellified $\mu$ ITIES array. The background-substracted CV profiles of insulin in the concentration range $1-15 \mu \mathrm{M}$ at the gellifed $\mu$ ITIES array are presented in Figure 3(A). The experiments were carried out under acidic conditions at $\mathrm{pH} 2$ (cell 2, Figure 1), conditions under which the insulin is fully protonated and cationic. An 
Revised Paper Ref.: C003323E. PCCP (RSC).

increase in the current on the forward sweep ( $\left.\mathrm{P} 1_{\text {insulin }}\right)$, associated with an increasing concentration of insulin in the aqueous phase solution, was observed at a very positive applied potential difference ( $\left.\Delta_{\beta}^{\alpha} \phi\right)$. This current response occurred close to the limit of the potential window, where the electrolyte transfer commences and, consequently, the shape of the resulting wave was difficult to ascertain. On the reverse sweep, a very broad peak with nonspecific adsorption/desorption features was observed ( $\left.\mathrm{P} 2_{\text {insulin }}\right)$. The currents on the forward sweeps of the background-subtracted CVs of insulin increased linearly with the concentration of insulin in the aqueous phase, whereas the reverse sweep saturated at $9 \mu \mathrm{M}$ of insulin in the aqueous phase (Figure 3(B)). This saturation event was previously seen for the extraction of oligopeptides. ${ }^{24}$ The current of the forward scan increased linearly with the concentration in the aqueous phase, as predicted by either the Randles-Sevcik equation ${ }^{36,} 37$ for the voltammetric peak current for linear diffusion systems or the Saito equation ${ }^{38}$ for the steady state current at an ultramicrodisk electrode. Irrespective of the diffusion mode, the current is concentration-dependent for both biomacromolecules.

The background-subtracted CV profiles of increasing concentrations of HEWL, in the range 0.5-13 $\mu \mathrm{M}$, at the gellifed $\mu$ ITIES array are illustrated in Figure 4(A). An increase in the current on the forward sweep $\left(\mathrm{P} 1_{\mathrm{HEWL}}\right)$, associated with an increasing concentration of HEWL in the aqueous phase solution, was observed at a very positive $\Delta_{\beta}^{\alpha} \phi$. The concentration dependence of the current is similar to that measured for insulin. On the reverse sweep an adsorption/desorption feature was evident ( $\left.\mathrm{P} 2_{\mathrm{HEWL}}\right)$. In comparison to insulin, this HEWL adorption/desorption peak was more compact and well defined. The maximum currents on the forward sweeps of the background-subtracted CVs of HEWL increased linearly with the 
Revised Paper Ref.: C003323E. PCCP (RSC).

concentration of HEWL in the aqueous phase, whereas the reverse sweep saturated at $7 \mu \mathrm{M}$ of HEWL in the aqueous phase (Figure 4(B)).

Comparison of the forward sweep currents for insulin and HEWL. A higher current was observed on the forward sweep for HEWL than for insulin, for equivalent concentrations in the aqueous phase. From studies of HEWL and insulin transfer at the ITIES, ${ }^{20,21}$ it is known that under more acidic conditions the biomacromolecules carry more positive charge than in neutral $\mathrm{pH}$ conditions, as reflected in the increased forward peak current.

The voltammetric current, exhibiting either steady state (limiting current) or transient behaviour (peak current), is proportional to the charge, $z_{\mathrm{j}}$, the diffusion coefficient, $D_{\mathrm{j}}$ and the bulk concentration of the species involved. The variables that influence the differences in behaviour between HEWL and insulin are the diffusion coefficients and the charges of the macromolecules in the aqueous phase at $\mathrm{pH} 2$. HEWL has a charge of $17+,{ }^{39}$ whereas insulin has a charge of $6+$ at $\mathrm{pH} 2 .{ }^{20}$ Insulin and HEWL have similar diffusion coefficients in the aqueous phase, with values of $1 \times 10^{-6} \mathrm{~cm}^{2} \mathrm{~s}^{-1}$ reported for both insulin ${ }^{40}$ and HEWL, ${ }^{41}$ as well as a value of $2.3 \times 10^{-6} \mathrm{~cm}^{2} \mathrm{~s}^{-1}$ for HEWL obtained from experiments at the ITIES. ${ }^{21}$ Therefore, based on its significantly larger charge, it is to be expected that HEWL will produce a greater current at the gellified $\mu$ ITIES arrays.

Two distinct possibilities for this charge transfer can be proposed. First, the biomacromolecules themselves are transferred across the ITIES on the forward sweep. The greater charge on the HEWL molecule causes the greater observed current on the forward sweep. Alternatively, as discussed previously for $\mathrm{Hb}$, insulin and $\mathrm{HEWL},{ }^{18-21}$ the biomacromolecule acts as a receptor at the ITIES and facilitates the transfer of the organic 
Revised Paper Ref.: C003323E. PCCP (RSC).

phase electrolyte anion $\mathrm{TPBCl}^{-}$into a protein layer on the aqueous side of the interface. Since $\mathrm{TPBCl}^{-}$is in concentration excess in the organic phase over the biomacromolecule in the aqueous phase, the biomacromolecule is the limiting component and consequently determines the current observed on the forward sweep. Therefore, with increasing concentration of the biomolecule, an increase of the ion transfer current is observed. Because HEWL is larger than insulin with the greater charge it has an increased ability to transfer organic anions, via an electrostatic attraction, across the interface, and subsequently complex these anions perhaps in a hydrophobic pocket of the biomacromolecule. For further investigation of the nature of the transfer reaction occurring in the presence of insulin or HEWL during the forward sweep, CV experiments with various potential scan rates were performed.

Scan rate studies of insulin and HEWL at a gellified $\mu$ ITIES array. A variable scan rate experiment involving $10 \mu \mathrm{M}$ of either insulin or HEWL at the gellified $\mu$ ITIES array was carried out at $\mathrm{pH}$ 2. At each scan rate, in the range $5-50 \mathrm{mV} \mathrm{s}^{-1}$, a blank $\mathrm{CV}$ was recorded in the absence of the biomacromolecules. The blank response was subtracted from the biomacromolecule response at each scan rate, and the resulting CVs are shown in Figure 5(A) for insulin and Figure 6(A) for HEWL. Plots of the current maxima of the backgroundsubtracted forward and backward potential sweeps versus the square root of the scan rate are shown in Figure 5(B) and Figure 6(B). Comparison of the CVs at different scan rates (Figures 5(A) and 6(A)) reveal that in the presence of insulin and HEWL, a steady-state current is not attained: instead, the current increases with increasing scan rate and a current peak is clearly detected at the lower scan rates. With increasing scan rate, the peak shifts to more positive potential and becomes masked by the onset of electrolyte transfer. 
Revised Paper Ref.: C003323E. PCCP (RSC).

The observation of current increase with increasing scan rate and the appearance of current peaks in the CVs both indicate that spherical diffusion of ions from the aqueous side to the inlaid micro-interfaces is unlikely during the forward sweep current. However, linear diffusion of the biomacromolecules from the aqueous side to the micro-interfaces could occur and this would explain the appearance of the peaks and the current dependence on the potential scan rate. This could happen only if the diffusion coefficients of HEWL or insulin in the aqueous phase were so small that, on the time scale of the experiment (typically $10-100$ s), the diffusion layer thickness is equal to or smaller than the critcal dimension (the radius) of the micropores $(26 \mu \mathrm{m})$. However, the diffusion coefficients ( ${ }^{\text {footnote }}{ }^{2}$ ) in aqueous solution for insulin $^{40}$ and HEWL ${ }^{42}$ are of the order of $1 \times 10^{-6} \mathrm{~cm}^{2} \mathrm{~s}^{-1}$ and the diffusion layer thickness, even at the highest scan rate used in the experiments, is ca. $30 \mu \mathrm{m}$, which exceeds the critical dimension of the micropore. Therefore, during the positive-going potential scan, the observed non-steady state current can only be explained by the interfacial transfer of the electrolyte anions, $\mathrm{TPBCl}^{-}$, from the organic phase to the aqueous side of the interface. The interfacial transfer of the anion is only possible in the presence of the positively charged HEWL or insulin ions adsorbed on the aqueous side of interface. Control experiments of repetitive CVs performed at the ITIES in both the presence and the absence of HEWL ${ }^{21}$ and $\mathrm{Hb}^{18}$ have also highlighted the fact that interfacial transfer of the anion is only possible in the presence of the positively charged biomacromolecule.

As mentioned earlier, the higher charge of HEWL, compared to insulin, results in transfer of more $\mathrm{TPBCl}^{-}$and therefore a higher current. However, the current increases with increasing concentration of both biomacromolecules. This could be explained by a desorption process

\footnotetext{
${ }^{2}$ We assume that the diffusion coefficient is not changed significantly by $\mathrm{pH}$, since protonation and any conformational change occurring with $\mathrm{pH}$ will have a minimal impact on the hydrodynamic radius of these biomacromolecules.
} 
Revised Paper Ref.: C003323E. PCCP (RSC).

after the biomolecules have formed a complex with the $\mathrm{TPBCl}^{-}$ion, and the vacant places are filled with uncomplexed biomolecules diffusing from the bulk solution. Also, the formation of multilayers ${ }^{18,21}$ at the interface with increasing biomolecule concentration supports the fact that the current increases with increasing biomolecule concentration. The current may be limited either by the adsorption/desorption process or by the diffusion of $\mathrm{TPBCl}^{-}$ions from the organic phase to the interface. On the one hand, $\mathrm{TPBCl}^{-}$is in concentration excess over the biomolecule concentration. On the other hand, the diffusion space of the anion is restricted by the micropore. Due to the viscosity of the gellified organic phase the diffusion coefficients of $\mathrm{TPBCl}^{-}$and of the biomolecules are of the same order of magnitude. ${ }^{23}$ As discussed, if diffusion of the biomolecules to the microinterface is controlling the overall observed current, then one would expect a steady-state limiting current, since spherical diffusion would dominate at the interface from the aqueous side. Clearly, however, steady-state voltammetry is not observed, thus the diffusion of $\mathrm{TPBCl}^{-}$ions from the organic phase to the interface is the current-carrying process.

In an effort to further elucidate the mechanistic role of $\mathrm{TPBCl}^{-}$, the influence of (BTPPA)(TPBCl) concentration on the $\mathrm{TEA}^{+}$voltammetric response was first investigated. However, it was found that $\mathrm{CV}$ of $50 \mu \mathrm{M} \mathrm{TEA}^{+}$transfer at the $\mu$ ITIES array using (BTPPA)(TPBCl) organogel concentrations lower than $10 \mathrm{mM}(1,0.5$, and $0.1 \mathrm{mM}$, respectively) produced distorted voltammograms due to increased resistance in the cell (not shown). As the $\mathrm{TPBCl}^{-}$concentration in the organogel phase was progressively decreased, the slope of the rising part of the $\mathrm{TEA}^{+}$steady-state response became shallower and the TEA ${ }^{+}$ half-wave transfer potential $\left(E_{1 / 2}\right)$ shifted towards higher potentials, a clear result of uncompensated resistance due to the diminished conductivity of the organo-gel phase. The heavy distortion of the $\mathrm{TEA}^{+} \mathrm{CV}$ prevented reliable analysis of the data based solely on the 
Revised Paper Ref.: C003323E. PCCP (RSC).

shape of the voltammogram. It was, thus, not feasible to conduct experiments with excess HEWL or insulin concentrations in the aqueous phase relative to the $\mathrm{TPBCl}^{-}$concentration in the organogel.

At this point, the complexity of the events occurring in the biomacromolecule detection process precludes definitive statements about the rate-determining step or kinetic limitations. The broad desorption peaks observed during the reverse scan suggest the desorption of multilayers or non-specifically adsorbed layers. To investigate further the adsorption behaviour of HEWL and insulin, CV experiments were performed in the presence of TEA ${ }^{+}$ and another, non-adsorbing, macromolecule.

\section{Electrochemical behaviour of TEA $^{+}$at a gellified $\mu$ ITIES array in the presence of an} adsorbed biomacromolecule and a non-adsorbed macromolecule, the dendrimer DABAM-4. HEWL and insulin are biomacromolecules with molecular weights of 14,600 and $5,733 \mathrm{~g} \mathrm{~mol}^{-1}$, respectively, and both are seen to form thick layers of white precipitate at the

interface after CV experiments. ${ }^{20,21}$ The electrochemistry of a much smaller molecule, DABAM-4, with a molecular weight of $316.53 \mathrm{~g} \mathrm{~mol}^{-1}$ was previously investigated ${ }^{43}$ at the ITIES at an aqueous phase $\mathrm{pH}$ of 5.5. The absence of a precipitate at the interface during these studies and the absence of any adsorption/desorption features in the voltammetric responses at the ITIES indicate that DAB-AM-4 undergoes simple ion transfer at the ITIES (in an identical manner to $\mathrm{TEA}^{+}$).

The electrochemical detection of DAB-AM-4 at pH 2 (cell 2, Figure 1) at the gellified $\mu$ ITIES array produced a sigmoidal response on the forward sweep (not shown) at close to the upper limit of the available potential window. A peak-shaped response, indicative of linear 
Revised Paper Ref.: C003323E. PCCP (RSC).

diffusion, was seen on the reverse sweep at a high $\Delta_{\beta}^{\alpha} \phi$ of $\sim 0.925 \mathrm{~V}$. After background subtraction, a peak was observed at a potential of $\sim 0.975 \mathrm{~V}$ on the forward sweep.

Interestingly, further evidence of the presence or absence of any adsorbed macromolecules at the $\mu$ ITIES was found on comparison of the background subtracted voltammetric responses of insulin, HEWL, and DAB-AM-4 after the addition of $15 \mu \mathrm{M} \mathrm{TEA}^{+}$to the aqueous phase (Figures 7(A), (B), and (C)). On transfer of the TEA ${ }^{+}$from the aqueous to the organogel phase in the presence of insulin or HEWL the expected steady-state response for TEA ${ }^{+}$transfer was distorted, seemingly perturbed by the presence of HEWL or insulin adsorbed at the interface. However, a TEA ${ }^{+}$steady-state response at $\Delta_{\beta}^{\alpha} \phi>0.7 \mathrm{~V}$ before the onset of macromolecule transfer into the organogel at ca. $0.9 \mathrm{~V}$, indicating an absence of any adsorbed material at the interface, was observed on the forward sweep in the presence of DAB-AM-4. The reverse sweep for $\mathrm{TEA}^{+}$in the presence of all three molecules was unaffected, due to the adsorbed layers being present on the aqueous side of the micro-interfaces and desorbed before the peak potential for the reverse-scan transfer of $\mathrm{TEA}^{+}$is reached.

\section{Conclusions}

The behaviour of insulin and HEWL at a gellified $\mu$ ITIES array was investigated by CV with a view to determining whether a similar reaction to that which occurs at larger liquid|liquid interfaces not stabilised by gels. It was found that the current of the positive-going voltammetric sweep was carried by $\mathrm{TPBCl}^{-}$ions, which transfer from the organogel to the aqueous side of the interface, and that this transfer was preceded by adsorption of the biomacromolecule at the aqueous side of the aqueous|organogel interface. The $\mathrm{TPBCl}^{-}$ transfer only occurs in the presence of either insulin or HEWL in the aqueous phase. These 
Revised Paper Ref.: C003323E. PCCP (RSC).

biomacromolecules are proposed to adsorb at the aqueous side of the interfaces and facilitate the transfer of $\mathrm{TPBCl}^{-}$.

This hypothesis of $\mathrm{TPBCl}^{-}$transfer is further corroborated by the linear dependence of the current maximum during the forward potential sweep on the square root of the potential scan rate, consistent with a linear diffusion of molecules to the interface. The diffusion field of $\mathrm{TPBCl}^{-}$is confined within the micropores used to define the $\mu$ ITIES and diffusion is essentially linear. This is in contrast to the scan rate independence shown at the same $\mu$ ITIES array for simple diffusion-controlled TEA ${ }^{+}$transfer.

Comparison of the electrochemical responses of the biomacromolecules insulin and HEWL with that of the much smaller dendrimer DAB-AM-4 in the presence of $\mathrm{TEA}^{+}$provided further evidence for adsorption of the biomacromolecules at the interface. The expected TEA ${ }^{+}$ steady-state response was distorted in the presence of insulin or HEWL on transfer from the aqueous to the organogel phase. But in the presence of DAB-AM-4, a steady-state response for $\mathrm{TEA}^{+}$transfer was obtained, indicating an absence of any blocking by adsorbed material at the interface. Furthermore, the reverse peak for $\mathrm{TEA}^{+}$, which is found at lower potentials than the desorption peaks of the macromolecules, was unaffected in the presence of all three macromolecules.

The results presented demonstrate that the mechanism of biomolecular detection at ITIES is complex but that the use of miniaturised ITIES can shed some light on the transport and transfer processes involved.

\section{Acknowledgements}


Revised Paper Ref.: C003323E. PCCP (RSC).

The support of Science Foundation Ireland (Grant number 07/IN.1/B967) and the Irish Research Council for Science, Engineering and Technology (Embark Postgraduate Research Scholarship Scheme, grant number RS/2005/122) are gratefully acknowledged.

\section{References}

1. $\quad$ D. W. M. Arrigan, Anal. Lett., 2008, 41, 3233-3252.

2. $\quad$ R. A. W. Dryfe, Advances in Chemical Physics, Vol 141, 2009, 141, 153-215.

3. F. Reymond, D. Fermin, H. J. Lee and H. H. Girault, Electrochim. Acta, 2000, 45, 2647-2662.

4. $\quad$ Z. Samec, Pure Appl. Chem., 2004, 76, 2147-2180.

5. $\quad$ Z. Samec, E. Samcova and H. H. Girault, Talanta, 2004, 63, 21-32.

6. $\quad$ P. Vanysek and L. B. Ramirez, J. Chil. Chem. Soc., 2008, 53, 1455-1463.

7. V. Beni, M. Ghita and D. W. M. Arrigan, Biosens. Bioelectron., 2005, 20, 2097-2103.

8. D. P. Zhan, S. N. Mao, Q. Zhao, Z. Chen, H. Hu, P. Jing, M. Q. Zhang, Z. W. Zhu and Y. H. Shao, Anal. Chem., 2004, 76, 4128-4136.

9. D. W. M. Arrigan, M. Ghita and V. Beni, Chemical Communications, 2004, 732-733.

10. Y. Chen, Y. Yuan, M. Q. Zhang, F. Li, P. Sun, Z. Gao and Y. H. Shao, Science in China Series B-Chemistry, 2004, 47, 24-33.

11. J. Langmaier, E. Samcova and Z. Samec, Anal. Chem., 2007, 79, 2892-2900.

12. Z. Samec, A. Trojanek, J. Langmaier and E. Samcova, Electrochem. Commun., 2003, 5, 867-870.

13. P. J. Rodgers, P. Jing, Y. Kim and S. Amemiya, J. Am. Chem. Soc., 2008, 130, 74367442.

14. A. Trojanek, J. Langmaier, E. Samcova and Z. Samec, J. Electroanal. Chem., 2007, 603, 235-242.

15. Y. Yuan and S. Amemiya, Anal. Chem., 2004, 76, 6877-6886.

16. J. A. Ortuno, A. Gil, C. Serna and A. Molina, J. Electroanal. Chem., 2007, 605, 157161.

17. J. A. Ortuno, C. Sanchez-Pedreno and A. Gil, Anal. Chim. Acta, 2005, 554, 172-176.

18. G. Herzog, V. Kam and D. W. M. Arrigan, Electrochim. Acta, 2008, 53, 7204-7209.

19. G. Herzog, W. Moujahid, J. Strutwolf and D. W. M. Arrigan, Analyst, 2009, 134, 1608-1613.

20. F. Kivlehan, Y. H. Lanyon and D. W. M. Arrigan, Langmuir, 2008, 24, 9876-9882.

21. M. D. Scanlon, E. Jennings and D. W. M. Arrigan, Phys. Chem. Chem. Phys., 2009, 11, 2272-2280.

22. R. Zazpe, C. Hibert, J. O'Brien, Y. H. Lanyon and D. W. M. Arrigan, Lab Chip, 2007, 7, 1732-1737.

23. J. Strutwolf, M. D. Scanlon and D. W. M. Arrigan, Analyst, 2009, 134, 148-158.

24. M. D. Scanlon, G. Herzog and D. W. M. Arrigan, Anal. Chem., 2008, 80, 5743-5749.

25. C. J. Collins and D. W. M. Arrigan, Anal. Chem., 2009, 81, 2344-2349.

26. A. A. Stewart, Y. Shao, C. M. Pereira and H. H. Girault, J. Electroanal. Chem., 1991, 305, 135-139.

27. M. H. M. Caçote, C. M. Pereira, L. Tomaszewski, H. H. Girault and F. Silva, Electrochim. Acta, 2004, 49, 263-270. 
Revised Paper Ref.: C003323E. PCCP (RSC).

28. H. Katano, H. Tatsumi and M. Senda, Talanta, 2004, 63, 185-193.

29. L. R. Wetter and H. F. Deutsch, J. Biol. Chem., 1951, 192, 237-242.

30. C. Tanford and J. Epstein, J. Am. Chem. Soc., 1954, 76, 2170-2176.

31. C. Tanford and J. Epstein, J. Am. Chem. Soc., 1954, 76, 2163-2169.

32. J. Gao, M. Mrksich, F. A. Gomez and G. M. Whitesides, Anal. Chem., 1995, 67, 30933100.

33. D. E. Anderson, W. J. Becktel and F. W. Dahlquist, Biochemistry, 1990, 29, 24032408.

34. G. Herzog, P. Eichelmann-Daly and D. W. M. Arrigan, Electrochem. Commun., 2010, 12, 335-337.

35. H. J. Lee, P. D. Beattie, B. J. Seddon, M. D. Osborne and H. H. Girault, J. Electroanal. Chem., 1997, 440, 73-82.

36. J. E. B. Randles, Trans. Faraday Soc., 1948, 44, 327-338.

37. A. Sevcik, Coll. Czech. Chem. Commun., 1948, 13, 348-377.

38. Y. Saito, Rev. Polarog. (Jpn), 1968, 15, 177-187.

39. S. Kuramitsu and K. Hamaguchi, J. Biochem., 1980, 87, 1215-1219.

40. H. Osamu, C. Sumio, S. Yuko, J. Kazuhiko, M. Kazuhiro and S. Toshinobu, J. Pharm. Pharmacol., 2004, 56, 1501-1507.

41. C. Mattisson, P. Roger, B. Jönsson, A. Axelsson and G. Zacchi, J. Chromatogr. B, 2000, 743, 151-167.

42. S. B. Dubin, N. A. Clark and G. B. Benedek, J. Chem. Phys., 1971, 54, 5158-\&.

43. A. Berduque, M. D. Scanlon, C. J. Collins and D. W. M. Arrigan, Langmuir, 2007, 23, 7356-7364. 


\section{Figure captions}

Figure 1: Electrochemical cells utilised in this study.

Figure 2: (A) Back-ground substracted CVs of $100 \mu \mathrm{M}_{\text {TEA }}^{+}$transfer across the $\mu$ ITIES array with increasing scan rates. Scan rates applied were 5 (black), 7.5, 10, 15, 20, and 25 (light grey) $\mathrm{mV} \mathrm{s}^{-1}$, as indicated by the dashed arrow. Electrochemical cell as detailed in cell 1 (Figure 1). Solid arrows indicate the direction of potential sweep. (B) Determining scan rate dependence or independence of the measured currents by plotting the current versus the square root of the scan rate for the forward and reverse sweeps.

Figure 3: (A) Back-ground substracted CVs of 1 (light grey), 2, 3, 5, 7, 9, 11, 13, and 15 (black) $\mu \mathrm{M}$ insulin, as indicated by the dashed arrow, at the gellified $\mu$ ITIES array. Electrochemical cell as detailed in cell 2 (Figure 1). Scan rate used was $5 \mathrm{mV} \mathrm{s}^{-1}$. Solid arrows indicate the direction of potential sweep. (B) The currents maxima of the forward $\left(\mathrm{P} 1_{\text {insulin }}\right)$ and reverse $\left(\mathrm{P} 2_{\text {insulin }}\right)$ sweeps for each concentration.

Figure 4: (A) Back-ground substracted CVs of 0.5 (light grey), 1, 2, 3, 5, 7, 9, 11, and 13 (black) $\mu \mathrm{M}$ HEWL, as indicated by the dashed arrow, at the gellified $\mu$ ITIES array. Electrochemical cell as detailed in cell 2 (Figure 1). Scan rate used was $5 \mathrm{mV} \mathrm{s}^{-1}$. Solid arrows indicate the direction of potential sweep. (B) The currents maxima of the forward $\left(\mathrm{P} 1_{\mathrm{HEWL}}\right)$ and reverse $\left(\mathrm{P} 2_{\mathrm{HEWL}}\right)$ sweeps for each concentration.

Figure 5: (A) Back-ground substracted CVs of $10 \mu \mathrm{M}$ insulin at potential scan rates of 5 (light grey), 10, 15, 20, 25, 35, and 50 (black) $\mathrm{mV} \mathrm{s}^{-1}$, as indicated by the dashed arrow, at the gellified $\mu$ ITIES array. Electrochemical cell as detailed in cell 2 (Figure 1). Solid arrows indicate the direction of potential sweep. (B) Peak currents versus the square root of the scan rate for the forward and reverse sweeps.

Figure 6: (A) Back-ground substracted CVs of $10 \mu \mathrm{M}$ HEWL at potential scan rates of 5 (light grey), 10, 15, 20, 25, 35, and 50 (black) $\mathrm{mV} \mathrm{s}^{-1}$, as indicated by the dashed arrow, at the gellified $\mu$ ITIES array. Electrochemical cell as detailed in cell 2 (Figure 1). Solid arrows indicate the direction of potential sweep. (B) Peak currents versus the square root of the scan rate for the forward and reverse sweeps.

Figure 7: Back-ground substracted CVs of (A) $15 \mu \mathrm{M}$ insulin (black) plus 5 (dark grey) and 10 (light grey) $\mu \mathrm{M} \mathrm{TEA}^{+}$, (B) $15 \mu \mathrm{M}$ HEWL (black) plus 5 (dark grey) and 15 (light grey) $\mu \mathrm{M} \mathrm{TEA}^{+}$, and (C) $20 \mu \mathrm{M}$ DAB-AM-4 (black) plus $15 \mu \mathrm{M} \mathrm{TEA}^{+}$(light grey) at the gellified $\mu$ ITIES array. Electrochemical cell as detailed in cell 2 (Figure 1). Scan rate used was $5 \mathrm{mV} \mathrm{s}{ }^{-}$ ${ }^{1}$. Solid arrows indicate the direction of potential sweep. 
Revised Paper Ref.: C003323E. PCCP (RSC).

\section{Figure 1:}

\section{Cell 1:}

$\mathrm{Ag}_{(\mathrm{s})}\left|{\mathrm{Ag} / \mathrm{AgCl}_{(\mathrm{s})}}^{10 \mathrm{mM}(\mathrm{BTPPA})(\mathrm{Cl})} \begin{array}{c}10 \mathrm{mM} \text { (BTPPA)(TPBCl) } \\ 10 \mathrm{mM} \mathrm{LiCl} \\ \text { (aq.) }\end{array}\right| \begin{gathered}10 \mathrm{mM} \mathrm{LiCl} \\ \text { Gellified 1,6-DCH } \\ \end{gathered}$

Cell 2:

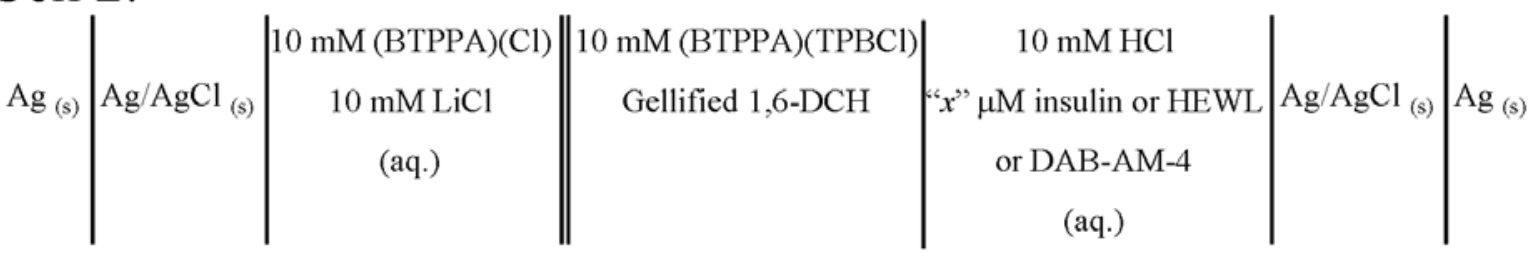


Revised Paper Ref.: C003323E. PCCP (RSC).

Figure 2:
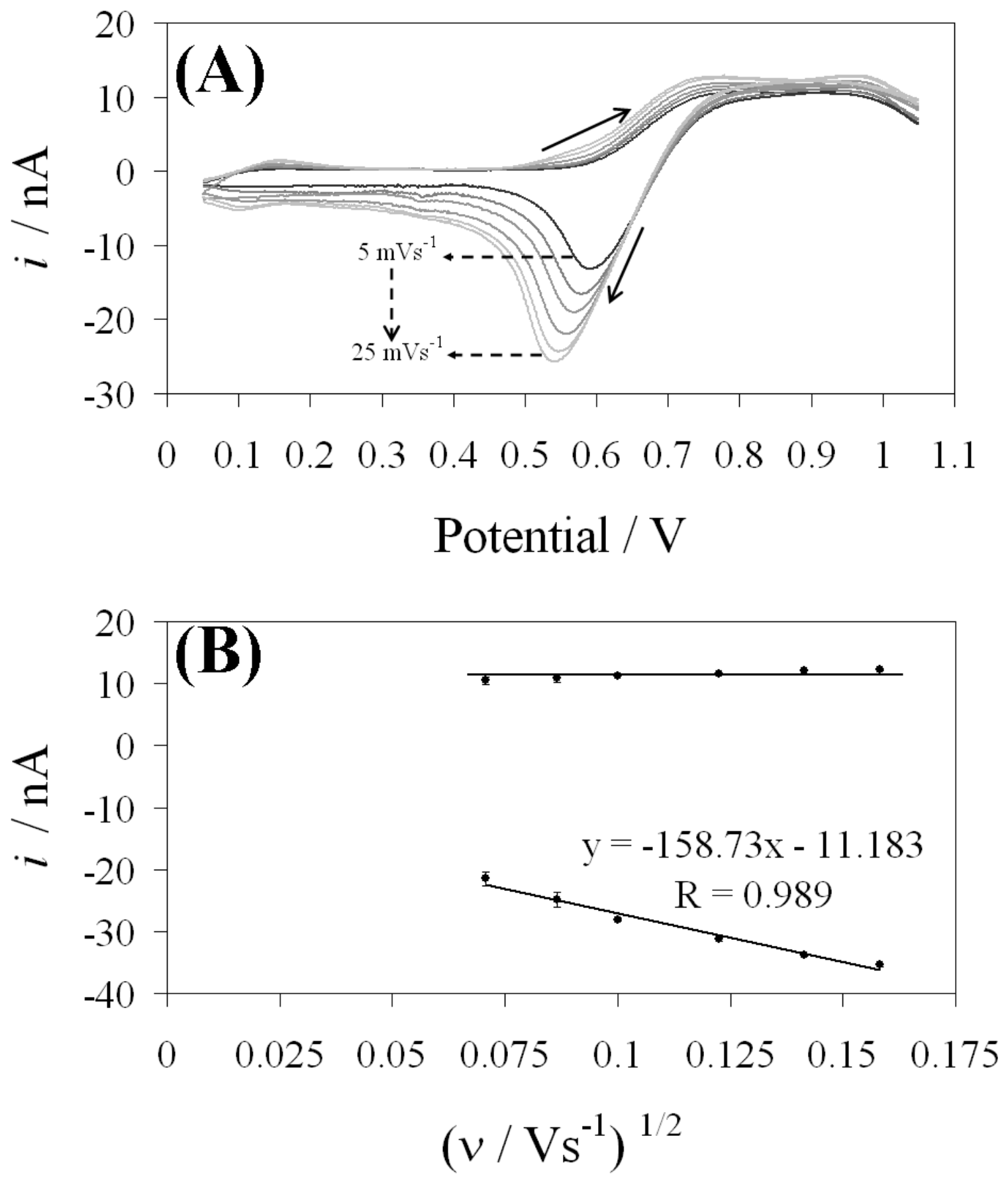
Revised Paper Ref.: C003323E. PCCP (RSC).

Figure 3:
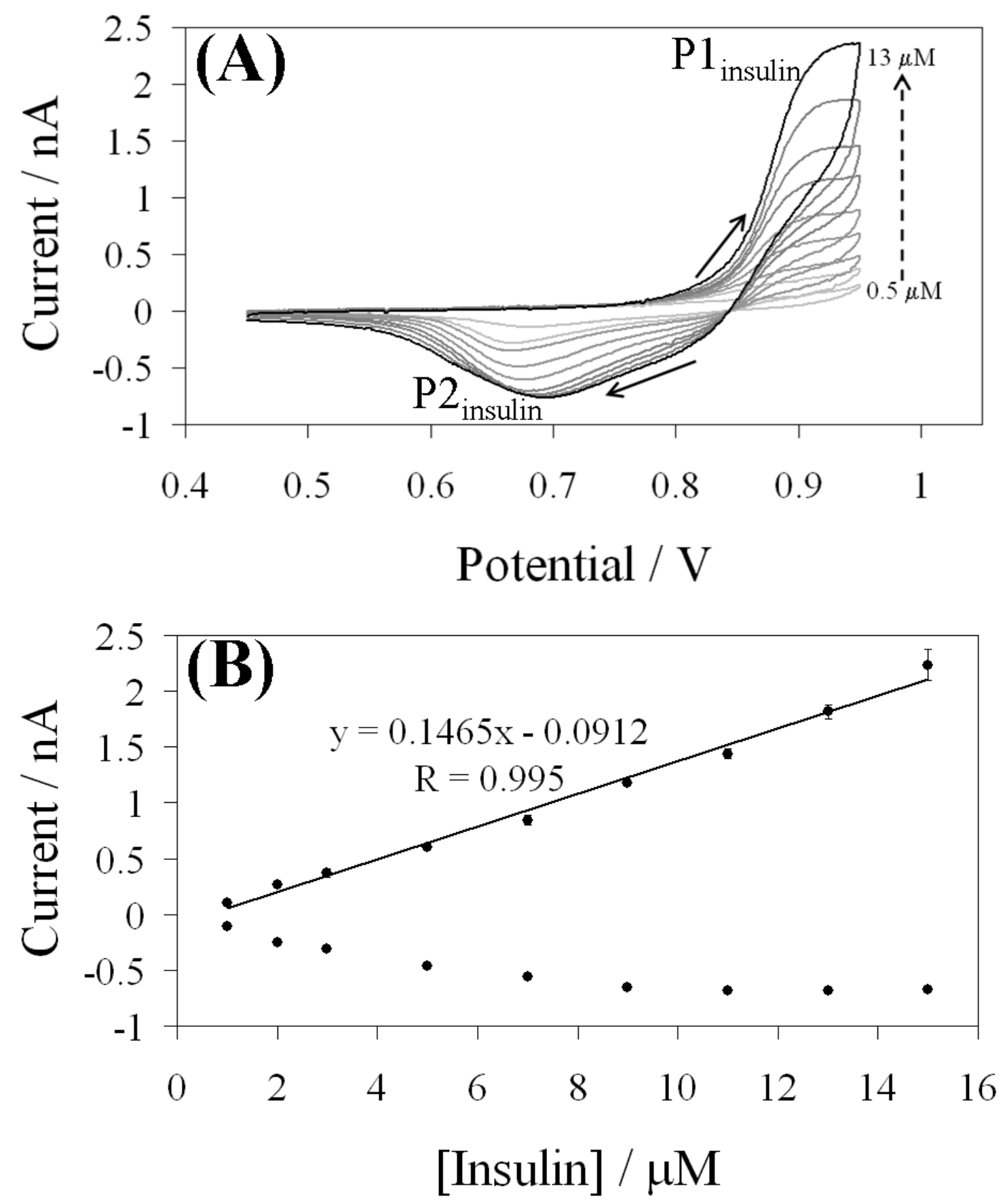
Revised Paper Ref.: C003323E. PCCP (RSC).

Figure 4:
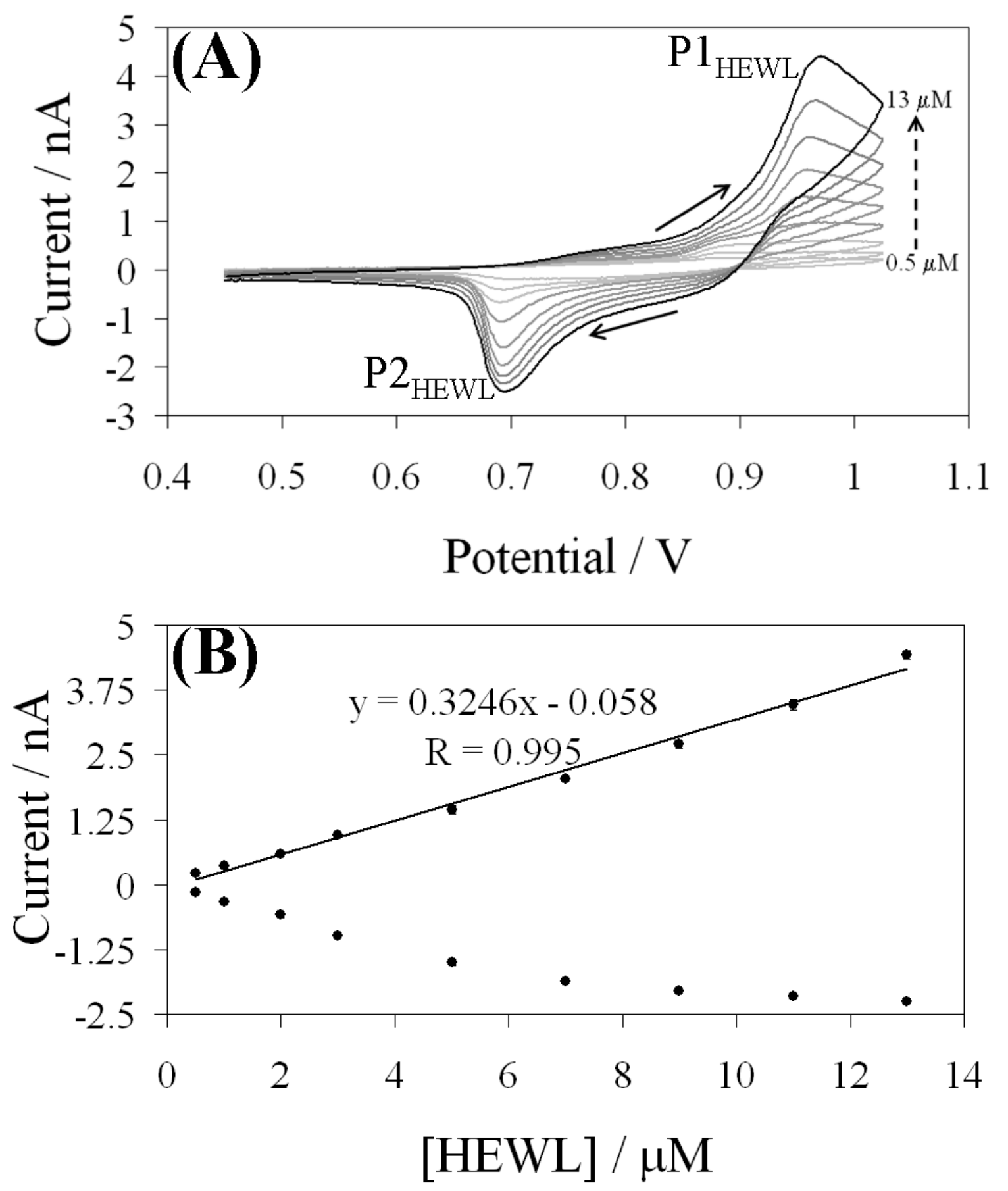
Revised Paper Ref.: C003323E. PCCP (RSC).

Figure 5:
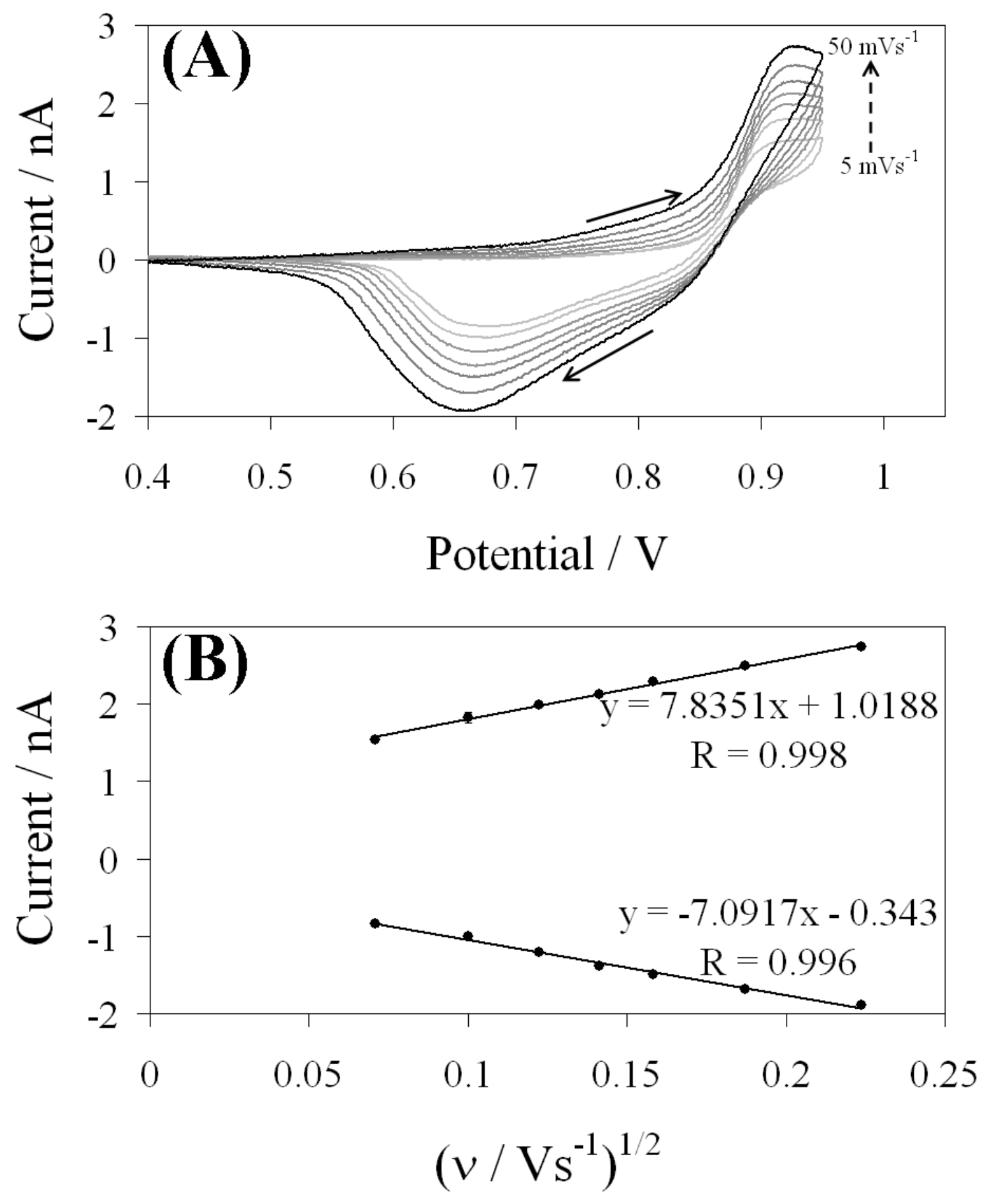
Revised Paper Ref.: C003323E. PCCP (RSC).

Figure 6:
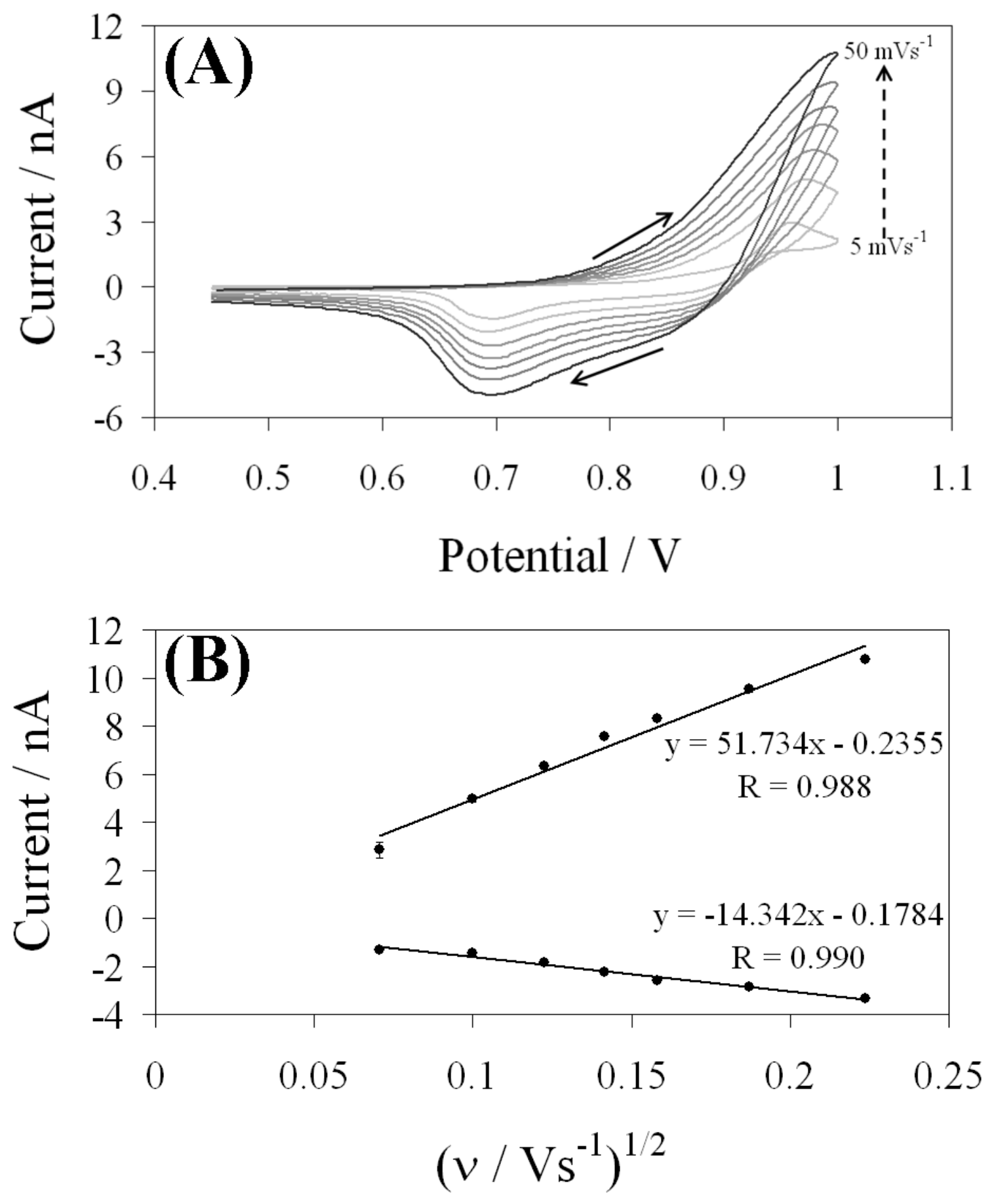
Revised Paper Ref.: C003323E. PCCP (RSC).

Figure 7: 
Revised Paper Ref.: C003323E. PCCP (RSC).
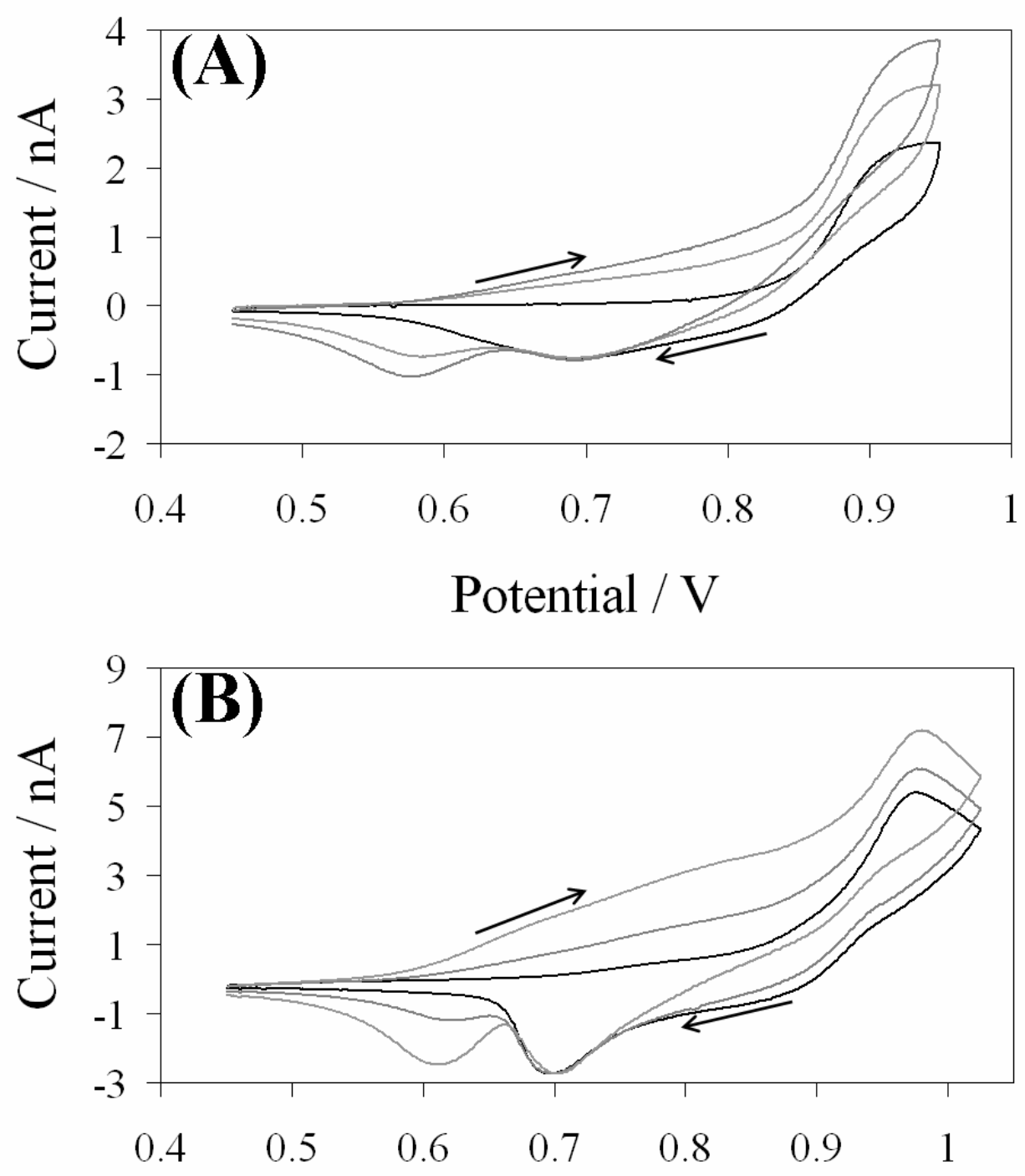

Potential / V

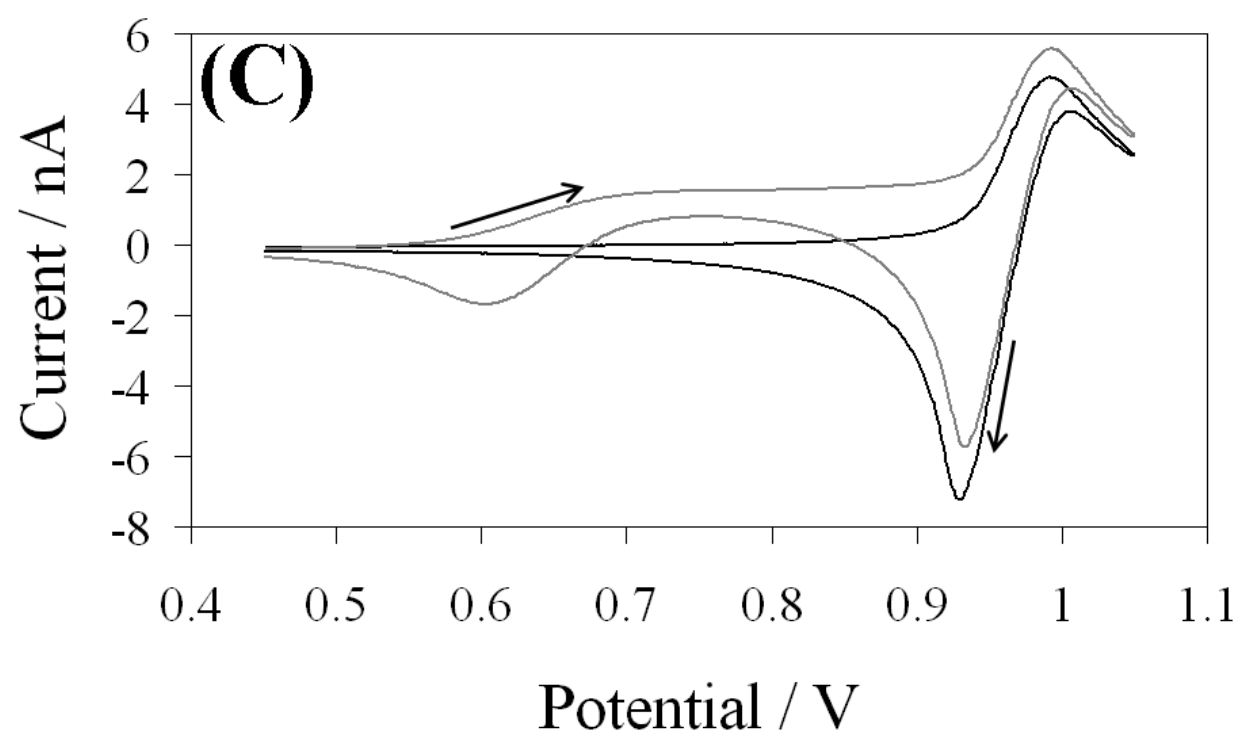

